ARTICLE OPEN

\title{
Logical design of oral glucose ingestion pattern minimizing blood glucose in humans
}

\author{
Masashi Fujii ${ }^{1,2,11}$, Yohei Murakami ${ }^{3}$, Yasuaki Karasawa ${ }^{4,5}$, Yohei Sumitomo ${ }^{2}$, Suguru Fujita ${ }^{2}$, Masanori Koyama ${ }^{6}$, Shinsuke Uda ${ }^{7}$,
} Hiroyuki Kubota ${ }^{7}$, Hiroshi Inoue ${ }^{8}$, Katsumi Konishi ${ }^{9}$, Shigeyuki $\mathrm{Oba}^{3}$, Shin Ishii $^{3,10}$ and Shinya Kuroda ${ }^{1,2,10}$

Excessive increase in blood glucose level after eating increases the risk of macroangiopathy, and a method for not increasing the postprandial blood glucose level is desired. However, a logical design method of the dietary ingestion pattern controlling the postprandial blood glucose level has not yet been established. We constructed a mathematical model of blood glucose control by oral glucose ingestion in three healthy human subjects, and predicted that intermittent ingestion 30 min apart was the optimal glucose ingestion patterns that minimized the peak value of blood glucose level. We confirmed with subjects that this intermittent pattern consistently decreased the peak value of blood glucose level. We also predicted insulin minimization pattern, and found that the intermittent ingestion $30 \mathrm{~min}$ apart was optimal, which is similar to that of glucose minimization pattern. Taken together, these results suggest that the glucose minimization is achieved by suppressing the peak value of insulin concentration, rather than by enhancing insulin concentration. This approach could be applied to design optimal dietary ingestion patterns.

npj Systems Biology and Applications (2019)5:31 ; https://doi.org/10.1038/s41540-019-0108-1

\section{INTRODUCTION}

In healthy people, blood glucose levels are stably maintained and show only a slight postprandial increase. ${ }^{1}$ However, massive postprandial increases in blood glucose levels emerge in patients with the type 2 diabetic mellitus (T2DM) and impaired glucose tolerance. ${ }^{2}$ This postprandial hyperglycemia requires prevention and treatment, because it is associated with increased risk of cardiac and cerebrovascular complications. ${ }^{3}$ Postprandial blood glucose originates from dietary carbohydrates. ${ }^{4}$ Some approaches to prevent postprandial hyperglycemia have thus far been reduction of dietary carbohydrate content, a change in the type of dietary carbohydrates, and ingestion of dietary fiber with meals. ${ }^{5}$ However, the ideal type of pattern for carbohydrate ingestion that minimize postprandial hyperglycemia is unknown.

Insulin, secreted from the pancreatic $\beta$ cells, performs a pivotal role in homeostatic regulation of blood glucose levels. Insulin acts on the target organs such as muscle and liver, to promote uptake of glucose from the blood and suppress hepatic glucose production. Consequently, insulin decreases blood glucose levels and promotes the rapid recovery of increase in postprandial blood glucose. As blood glucose levels decrease, insulin secretion also decreases. Thus, the blood glucose level is maintained within a narrow normal range by the feedback relationship between blood glucose and insulin. ${ }^{6}$
Although insulin secretion is regulated mainly by blood glucose, it is also regulated by a family of circulating hormones called incretins. ${ }^{7}$ Incretins are hormones secreted from the gastrointestinal tract upon food ingestion, these hormones act on pancreatic $\beta$ cells to promote insulin secretion. Gastric inhibitory polypeptide (GIP) and glucagon-like peptide-1 (GLP-1) are incretins. ${ }^{7-10}$ GIP is secreted from $\mathrm{K}$ cells of the upper small intestine; ${ }^{11,12}$ GLP-1 is secreted from $L$ cells of the lower small intestine. ${ }^{13,14}$ Orally ingested glucose promotes incretin secretion into the small intestine, where it is absorbed and enters the blood. Blood glucose and incretin act cooperatively on pancreatic $\beta$ cells to promote insulin secretion and increase circulating insulin levels. ${ }^{15}$

Postprandial hyperglycemia is identified with an oral glucose tolerance test (OGTT), in which a subject's ability to tolerate a glucose load (glucose tolerance) is evaluated by measuring blood glucose level after an overnight fast and again $2 \mathrm{~h}$ after a 75 -g oral glucose load. ${ }^{16}$ Using time course data of glucose and insulin in the blood during the OGT, many mathematical models have quantitatively evaluated the relationship between the blood glucose and insulin in humans. ${ }^{17-26}$ These models consist of blood glucose and insulin, but not incretins. ${ }^{17,18,27-29}$ Other mathematical models incorporate the incretins. ${ }^{24,26,30-32}$ In some models, blood glucose and incretin act independently on insulin secretion during the OGT; ${ }^{30-32}$ in others, blood glucose and incretin act cooperatively. ${ }^{24,26}$ The effective action of incretins on

\footnotetext{
${ }^{1}$ Molecular Genetic Research Laboratory, Graduate School of Science, The University of Tokyo, Tokyo 113-0033, Japan; ${ }^{2}$ Department of Biological Sciences, Graduate School of

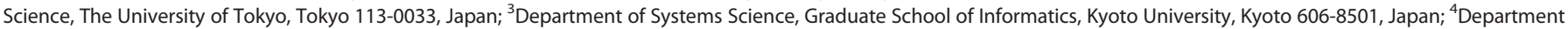

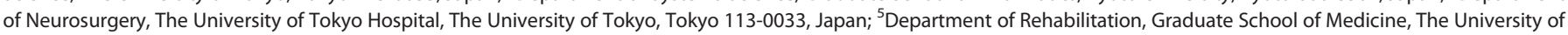
Tokyo, Tokyo 113-0033, Japan; ${ }^{6}$ Department of Mathematics, Graduate School of Science and Engineering, Ritsumeikan University, Shiga 525-8577, Japan; ${ }^{7}$ Division of Integrated Omics, Research Center for Transomics Medicine, Medical Institute of Bioregulation, Kyushu University, Fukuoka 812-8582, Japan; ${ }^{8}$ Metabolism and Nutrition Research Unit, Institute for Frontier Science Initiative, Kanazawa University, Ishikawa 920-8640, Japan; ${ }^{9}$ Faculty of Computer and Information Sciences, Hosei University, Tokyo 184-8584, Japan; ${ }^{10} \mathrm{CREST}$, Japan Science and Technology Agency, Tokyo 113-0033, Japan; ${ }^{11}$ Present address: Department of Integrated Sciences for Life, Graduate School of Integrated Sciences for Life, Hiroshima University, Hiroshima 739-8526, Japan

Correspondence: Shinya Kuroda (skuroda@bs.s.u-tokyo.ac.jp)

These authors contributed equally: Masashi Fujii, Yohei Murakami, Yasuaki Karasawa
}

Received: 5 March 2019 Accepted: 6 August 2019

Published online: 02 September 2019 
the insulin secretion in mathematical models remains to be determined.

One application of mathematical models is the ability to make prediction. Published mathematical models of blood glucose and insulin have been used to predict blood glucose levels after glucose administration. We require a solution of a pair of forward and inverse problems to obtain an optimal design of input pattern. Firstly, we need a dynamics model to predict the temporal pattern as a consequence of a given input pattern. This mode of prediction is a forward problem: The prediction is an "output pattern" related to the input pattern. Secondly, optimal input pattern should be determined so as to minimize the outcome that is defined as an arbitrarily given objective function of the predicted output pattern. This mode of prediction is an inverse problem: The prediction is an "input pattern" that produces an optimal output pattern. There are many established methods that use complex ordinary differential equations to solve the forward problem of predicting output patterns, but few methods exist to solve the inverse problem of predicting input patterns. Recently, we proposed a mathematical framework to estimate an input pattern that produces a defined output pattern. $^{33}$

Here, we constructed mathematical models with either glucoseindependent and/or glucose-cooperative roles of incretins on insulin secretion. We used the models to predict an optimal glucose ingestion pattern that controls blood glucose level. Because blood glucose level is the output pattern, this represents using the model to solve an inverse problem. We measured blood glucose, insulin, GIP, and GLP-1 before and after oral glucose ingestion with different doses and ingestion durations for three subjects. As a forward problem, we constructed a mathematical model of blood glucose (output) in response to orally ingested glucose (input) for each subject. As an inverse problem, we optimally designed glucose ingestion pattern that minimizes the peak value of blood glucose level for each subject. Note that the terms forward and inverse problems have limited meaning in some research fields, but we use the terms forward and inverse problems in a more general sense. In other words, assuming that the problem commonly utilized in the field of systems biology, i.e., estimating model parameters that fit the data, is a forward problem, predicting an input pattern that realizes a time series satisfying a certain objective function from model and parameters can be regarded as an inverse problem. Each subject had an optimized pattern of ingestion that was intermittent. We validated blood glucose level by the predicted intermittent ingestion pattern for each subject and found that the intermittent ingestion pattern decreased the peak value of blood glucose level compared with the blood glucose levels that occurred with bolus or 1-h-continuous ingestion patterns. Thus, we provide the logical design of oral glucose ingestion pattern that minimizes the peak value of blood glucose level in humans, using an approach of combination of a forward and an inverse problems, which can be widely applied to design optimal dietary ingestion patterns for human health.

\section{RESULTS}

Measurement of blood glucose and blood hormones before and after oral glucose ingestion

To obtain the data for developing the model, we monitored the effect of ingestion of different amounts of glucose in different temporal patterns of ingestion on blood glucose and hormone levels (Fig. 1). In six separate experiments, the three healthy volunteers either rapidly consumed one of three doses of glucose $(25,5$, and $75 \mathrm{~g})$ or consumed the glucose over $2 \mathrm{~h}$ (see Methods). The rapid ingestion paradigm is referred to as bolus ingestion and the slow ingestion paradigm as $2 \mathrm{~h}$-continuous ingestion. Prior to

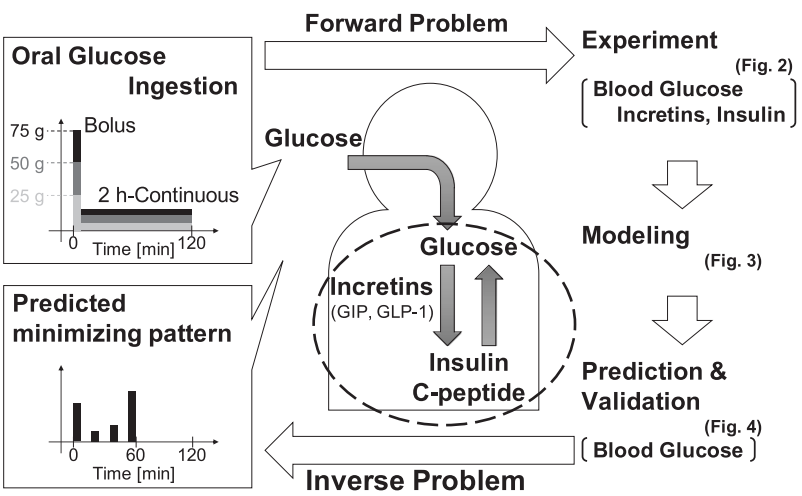

Fig. 1 Study diagram. Three subjects orally ingested glucose with three doses 75, 50, and $25 \mathrm{~g}$ in two durations of bolus and $2 \mathrm{~h}$ continuous ingestion. Time course data of blood glucose level, insulin level, C-peptide level, GIP level, and GLP-1 levels were obtained (Fig. 2). We constructed models of the dynamics of these blood hormones and glucose for each subject as a forward problem (Fig. 3). Using the models, we predicted the glucose minimization pattern, the glucose ingestion pattern minimizing the peak value of blood glucose level for the ingestion of $50 \mathrm{~g}$ glucose within $60 \mathrm{~min}$ as an inverse problem, and validated the pattern experimentally (Fig. 4)

glucose ingestion and after glucose ingestion, we measured levels of blood glucose, insulin, C-peptide, intact GIP (designated GIP hereafter), and intact GLP-1 (designated GLP-1 hereafter) (see Methods).

With any ingestion pattern, the temporal pattern of each molecule exhibited a transient increase that returned to baseline within $4 \mathrm{~h}$ (Fig. 2). For bolus ingestion, the blood glucose and other blood hormones reached similar peak values for each dose of ingested glucose (Fig. 2a, c, e, g, i). For the 2-h-continuous ingestion, blood glucose and other blood hormones showed increasing peak values with increasing doses of ingested glucose (Fig. 2b, d, f, h, j). A consistent difference between bolus and continuous ingestion was that in the bolus ingestion case, with increasing doses of glucose, the time when blood glucose and hormones began to decrease and time to return to baseline become more delayed. In contrast, for 2-h-continuous ingestion, the time when blood glucose and other hormones began to decrease, and the time when all returned to the basal level were similar regardless of dose of ingested glucose. Subjects \#2 and \#3 showed similar responses to subject \#1 by bolus and 2-hcontinuous ingestion, except for GLP-1 (Supplementary Fig. S1). GLP-1 for only $75 \mathrm{~g}$ bolus ingestion for subject \#1 showed a high transient peak, but that for subjects \#2 and \#3 did not.

\section{Mathematical model of blood glucose control}

As a solution to the forward problem, we constructed a mathematical model of blood glucose control that fits time course data of blood glucose and hormones. We constructed a mathematical model from ordinary differential equations (Fig. 3a, Supplementary Table S1, see Methods). Because of possible alternative mechanisms of actions of GIP and GLP-1 on insulin secretion, ${ }^{24,26,30-32}$ we constructed multiple alternative models in which the GIP or GLP-1 or both have independent actions or cooperative actions with blood glucose to promote insulin secretion (Fig. 3a, Supplementary Table S1, see Methods). We estimated parameters of each model for each subject separately to fit time course data of blood glucose and hormones. We selected the best model of blood glucose control for each subject by Akaike Information Criterion (AIC) (see Methods). Note that the GIP concentration of subject \# 1 is lower than that of the other subjects in all experiments, and the GLP-1 concentration is also 

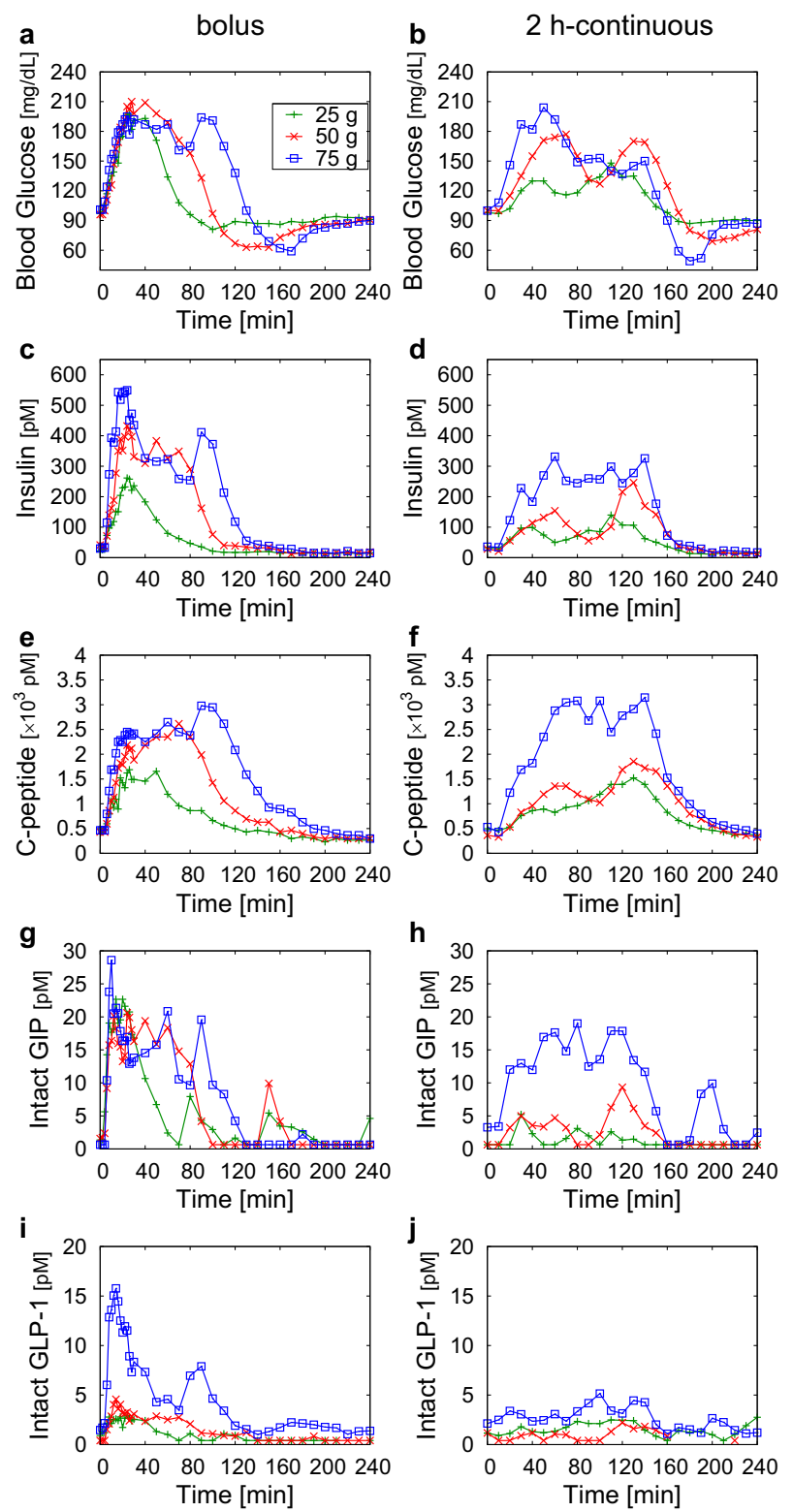

Fig. 2 Time course data of blood glucose level and blood hormones in subject \#1 by glucose ingestion. Time course data of blood glucose level and blood hormones in subject $\# 1$ by glucose ingestion. a, b Blood glucose. c, d Insulin. e, f C-peptide. g, h Intact GIP. i, j Intact GLP-1. a, c, e, g, i Bolus ingestion. $\mathbf{b}, \mathbf{d}, \mathbf{f}, \mathbf{h}, \mathbf{j} 2 \mathrm{~h}$ continuous ingestion. The doses are indicated in panel a

higher. Nevertheless, every subject has the similar insulin peaks, indicating that how incretin works on insulin secretion must be different between individuals. In addition, in each subject, GIP and GLP-1 showed a peak at about 5-10 min in the bolus ingestion, and then gradual decrease (Fig. 2). Therefore, the time courses of GIP and GLP-1 show a high correlation, and overfitting caused by multicollinear may occur. Therefore, the dominant effect of GIP and GLP-1 on insulin secretion should be considered through the model selection, which identifies the model reflecting the effective dynamics of blood glucose levels without overfitting.

The selected models were the same for subjects \#1 and \#3, but different from the model for subject \#2 (Supplementary Table S2, S3). In the models of subject \#1 and \#3, cooperative action by blood glucose and GIP was selected, indicating that insulin secretion did not depend on GLP-1. In the model of subject \#2, the independent action of GIP and Cooperative action by blood glucose and GLP-1 were selected. In each subject model, time course data of each blood glucose and hormones were approximately reproduced (Fig. 3b, Supplementary Figs. S2 and S3, and Supplementary Table S4).

Optimization and validation of glucose ingestion pattern that minimizes peak value of blood glucose level

Using mathematical, we tackled the inverse problem of predicting an optimal input pattern that optimally controls the output pattern. Here, input and output patterns are, specifically, time courses of oral glucose ingestion and blood glucose level, respectively. The optimality of the output pattern is defined as an objective function that is a function of the output pattern, typically the peak value of blood glucose level. First, we optimized the glucose ingestion pattern for each subject that minimized the typical objective function. Hereafter, we designate the optimized patterns minimizing objective function as the glucose minimization pattern. We searched the solution under the following restrictions; total $50 \mathrm{~g}$ of glucose should be ingested within $60 \mathrm{~min}$, glucose is ingested every $5 \mathrm{~min}$, at least $1 \mathrm{~g}$ is ingested at $0 \mathrm{~min}$ and the remaining $49 \mathrm{~g}$ of glucose is distributed between 0 and $60 \mathrm{~min}$ Because the combination of glucose ingestion patterns is enormous $(62 ! /(49 ! 13 !))$, we obtained an optimal ingestion pattern using an evolutionary programing-based optimization algorithm (see Methods). ${ }^{34}$ The glucose minimization patterns for the three subjects were designed with the aboveexplained method and shown in Fig. 4a, red line.

The optimized glucose minimization pattern of the subject \#1 appeared to be an intermittent pattern with 30 -min intervals with most glucose ingested at $0 \mathrm{~min}(17 \mathrm{~g})$ and $60 \mathrm{~min}(23 \mathrm{~g})$, and smaller amounts ingested at $30 \mathrm{~min}(8 \mathrm{~g})$ and $35 \mathrm{~min}(2 \mathrm{~g})$ (Fig. 4a, red line; Supplementary Table S5). This pattern was different from bolus and 1-h-continuous ingestions. The predicted blood glucose achieved with the glucose minimization pattern showed a bimodal temporal pattern with peaks from $\sim 25-50 \mathrm{~min}$ and at $\sim 80$ min (Fig. 4b, red line).

The optimized glucose minimization patterns of subjects \#2 and \#3 appeared to be intermittent patterns similar to the pattern of the subject \#1 (Fig. 4a, red line; Supplementary Table S5). Compared with subject \#1, for subjects \#2 and \#3, the optimized pattern of ingestion had some notable differences: Ingestion amount of glucose at 0 min was less, the number of time points at 30-min the intermittent period during which glucose was ingested was larger, and the ingestion amount of glucose at 60 min was larger. The predicted blood glucose level achieved with the glucose minimization pattern for subjects \#2 and \#3 showed a similar bimodal pattern to that for subject \#1 (Fig. 4b, red line).

We also compared the simulated blood glucose levels produced with the glucose minimization pattern with those simulated for bolus or 1-h-continuous ingestion of $50 \mathrm{~g}$ of glucose. The predicted glucose minimization pattern produced a lower peak value of blood glucose level than either simulations of bolus or 1$\mathrm{h}$-continuous ingestion using the subject-specific models (Fig. 4b).

We validated the predicted blood glucose levels produced with the glucose minimization patterns for each subject. Each subject ingested glucose according to their specific optimized glucose minimization pattern (Supplementary Table S5), and blood glucose levels were measured. (Fig. 4c, red line). The peak value of blood glucose level produced by ingestion according to the glucose minimization pattern in each subject was less than those produced by bolus and 1-h-continuous ingestion (Fig. 4c, Supplementary Table S6). All subjects exhibited bimodal temporal patterns of blood glucose level. These experimental results are consistent with the predictions except that first peak in blood glucose level at $\sim 30 \mathrm{~min}$ was lower than the second peak at $\sim 80$ min for subjects \#1 and \#2 and the peak in blood glucose was 


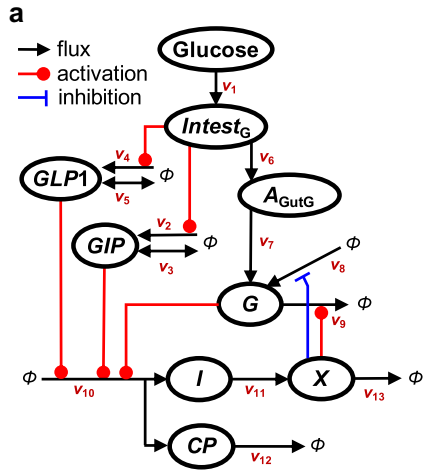

b
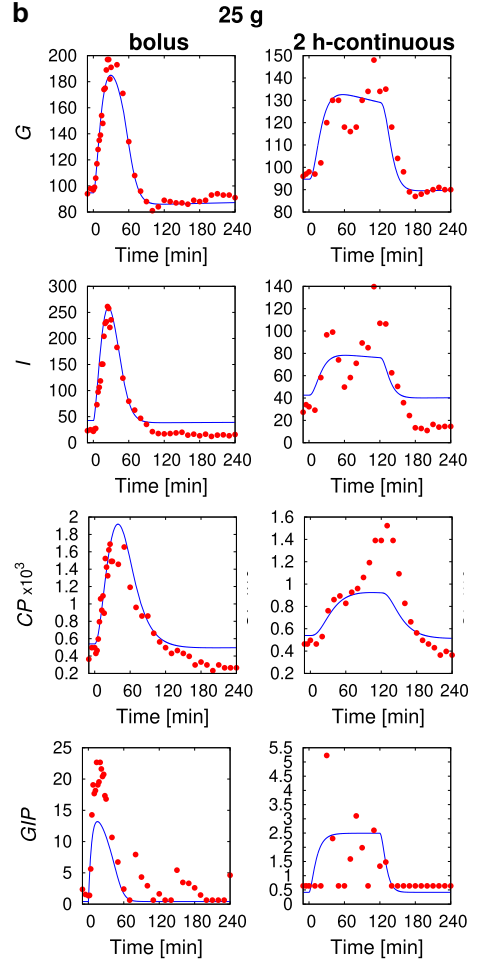
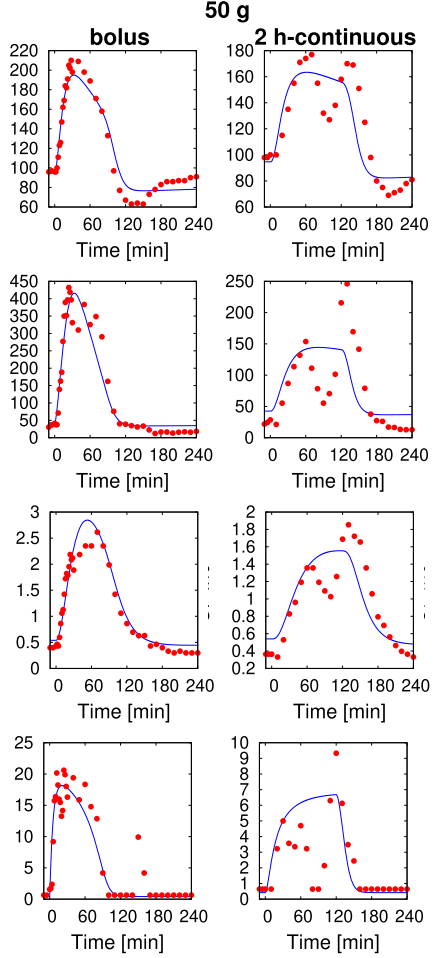

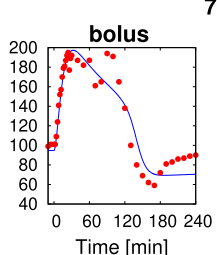

$75 \mathrm{~g}$
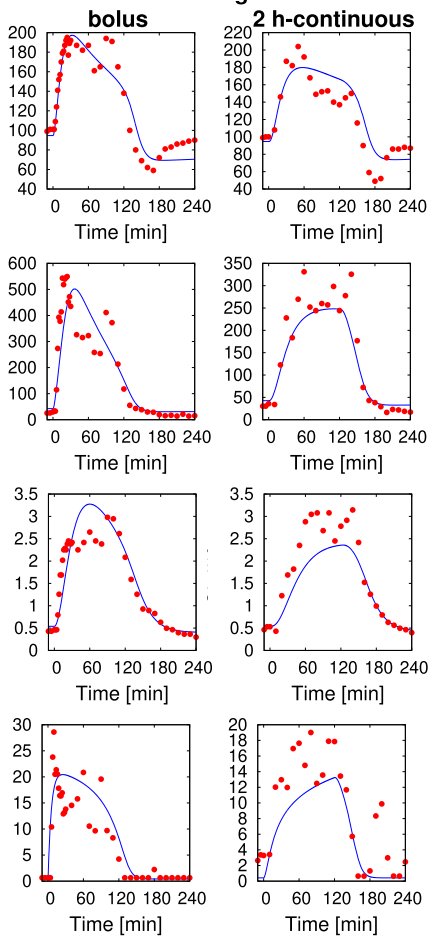

Fig. 3 The blood glucose control model. a Model diagram. The letters in the circle indicate the variables of the model, the arrows indicate the flow of molecules, the red lines indicate activation, and the blue line indicates inhibition (see Methods). The best fitting models for subjects \# 1 and \#3 lack the GLP1 components. b Temporal patterns of hormones. The blue lines indicate the temporal patterns of simulations, and the red circles indicate the time course data of experiments. The dose and ingestion pattern are indicated at the top

delayed from the prediction for subject \#3. The peak value of insulin concentration by the glucose minimization pattern was lower than that by the bolus and 1-h-continuous ingestion (see Fig. 4d). This result indicates that the glucose minimization is not achieved by enhancing insulin concentration.

Next, to verify the possibility that glucose minimization is because of larger insulin secretion, we set the objective function as the peak value of insulin and obtained the pattern that minimizes the peak value of insulin for each subject (Fig. 4a, blue line). Hereafter, we designate this pattern as the insulin minimization pattern. The optimized insulin minimization pattern of the subject \#1 appeared to be an intermittent pattern with 30min intervals with most glucose ingested at 0 min $(21 \mathrm{~g})$ and $60 \mathrm{~min}(19 \mathrm{~g})$, and smaller amounts ingested at $15 \mathrm{~min}(1 \mathrm{~g})$, $25 \mathrm{~min}(3 \mathrm{~g}), 30 \mathrm{~min}(2 \mathrm{~g})$, and $35 \mathrm{~min}$ (4 g) (Fig. 4a, blue line; Supplementary Table S5). This pattern was different from bolus and 1-h-continuous ingestions and similar intermittent pattern to glucose minimization pattern (Fig. 4a). The both of predicted blood glucose level and insulin concentration achieved with the insulin minimization pattern showed bimodal temporal patterns with peaks from $\sim 15-50 \mathrm{~min}$ and at $\sim 80 \mathrm{~min}$, respectively (Fig. $4 \mathrm{~b}$ and $d$, blue line).

The optimized insulin minimization patterns of subjects \#2 and \#3 appeared to be intermittent patterns similar to the pattern of the subject \#1 (Fig. 4a, blue line; Supplementary Table S5). The both of predicted blood glucose level and insulin concentration achieved with the insulin minimization pattern for subjects \#2 and \#3 showed a similar bimodal pattern to that for subject \#1 (Fig. 4b and $d$, blue line). Compared with glucose minimization pattern, the optimized insulin minimization pattern of ingestion was a similar intermittent pattern for each subject, but had some notable differences: Ingestion amount of glucose at 0 min was larger, and the ingestion amount of glucose at 60 min was less. Although there is the quantitative difference of ingestion dose, the intermittent glucose ingestion pattern decreases the peak values of blood glucose level and insulin concentration for all subject. These suggest that the glucose minimization is achieved by suppressing the peak value of insulin concentration, rather than by enhancing insulin concentration.

\section{DISCUSSION}

Prediction and validation of glucose ingestion patterns that minimize the peak value of blood glucose level and insulin concentration

In this study, as a forward problem, we constructed a mathematical model of the change in blood glucose from time course data of blood glucose and hormones in blood during and following oral glucose ingestion with various doses and durations in human subjects (Figs. 2, 3). The increase of postprandial blood glucose may cause angiopathy, such as macroangiopathy, as described in the Introduction. Then, using this model, as an inverse problem, we optimized glucose ingestion patterns that minimize the peak value of blood glucose level and validated these patterns with the human subjects by experiments (Fig. 4). The glucose minimization pattern was an intermittent pattern different from both the bolus ingestion and the continuous ingestion. This intermittent ingestion pattern was intuitively not obvious. However, we discovered the pattern using this approach of both constructing a mathematical model as a forward problem and optimizing input pattern from the model as an inverse problem. Although the best fitting model for each subject had important differences in the roles of the blood hormones, the intermittent pattern as an optimal ingestion pattern to minimize peak value of blood glucose level was common to all three subjects, suggesting that the glucose minimization pattern is robust to these differences in the model. Although we determined that the duration of the intermittent period was a key parameter controlling the glucose 


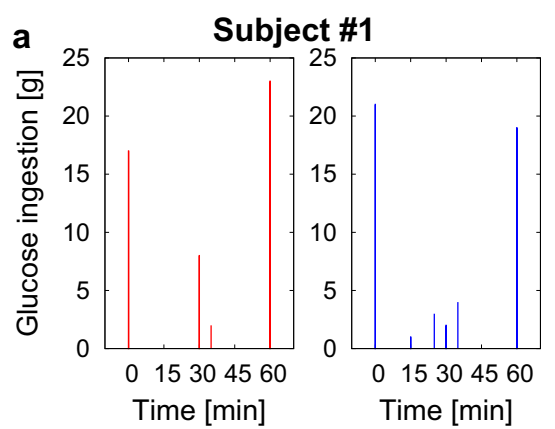

b
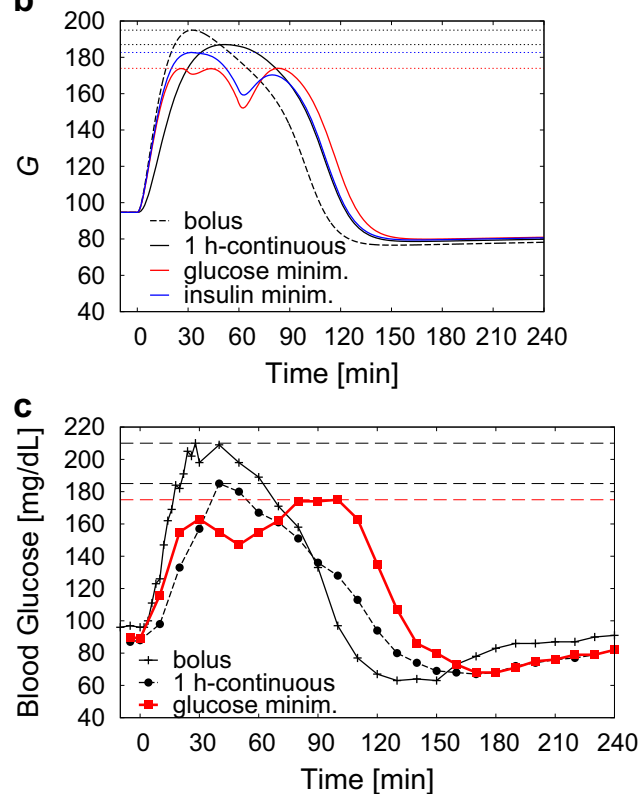

d

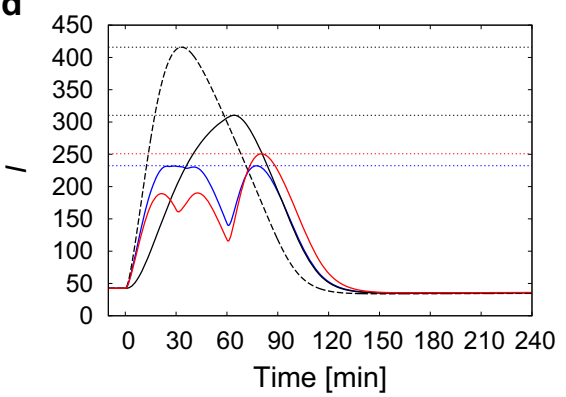

Subject \#2
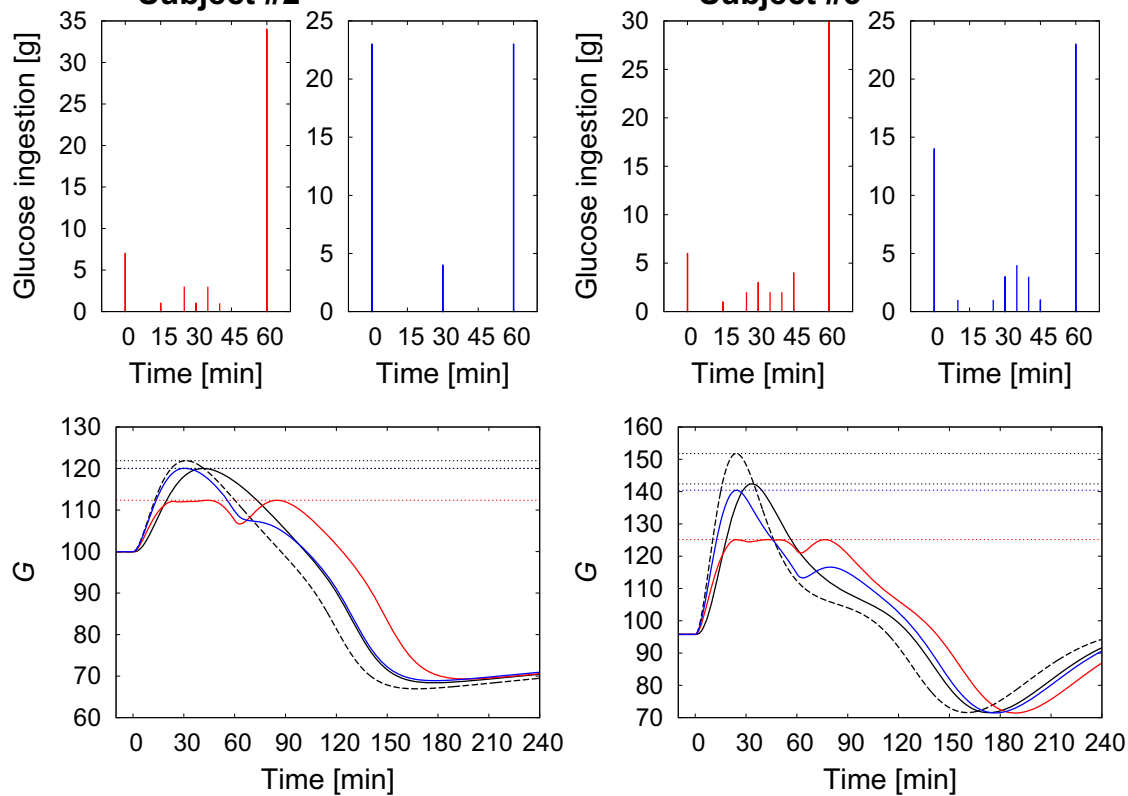

Subject \#3

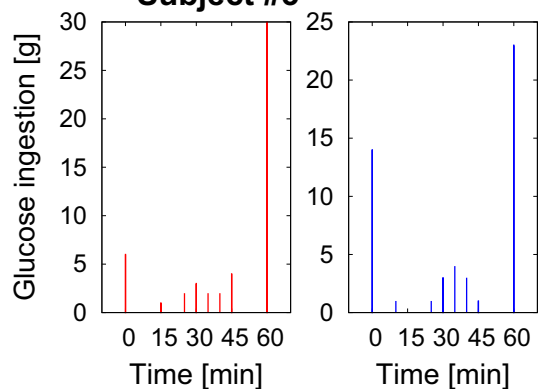

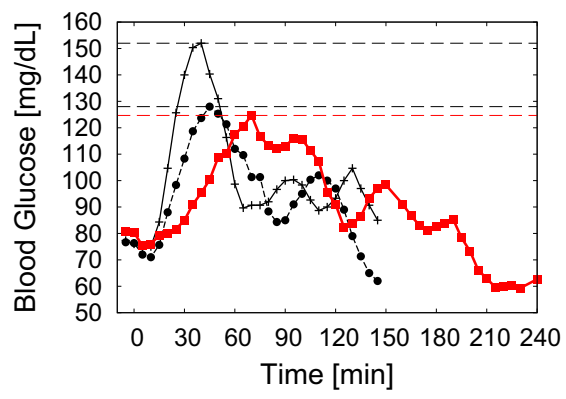

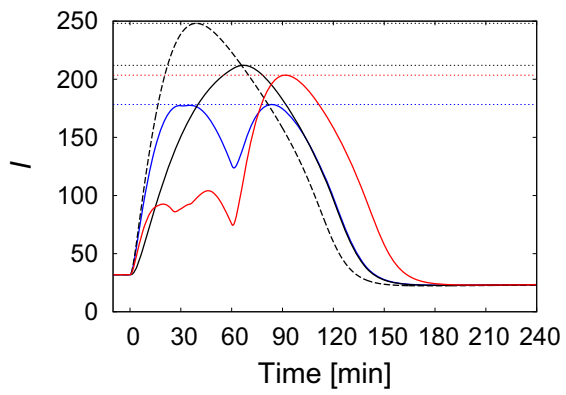

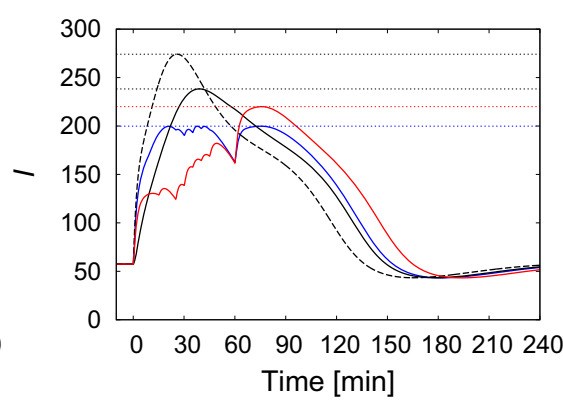

Fig. 4 Optimal patterns minimizing the peak values of blood glucose level or insulin concentration. a Glucose minimization pattern (left) and insulin minimization pattern (right) for glucose ingestion that minimizes the peak value of blood glucose level in subject \#1, \#2, and \#3. b Temporal patterns of blood glucose simulated from ingestion of glucose according to the glucose minimization pattern (red line), the insulin minimization pattern (blue line), bolus ingestion (black solid line), or $1 \mathrm{~h}$-continuous ingestion (black broken line). The peak values achieved for each ingestion pattern are marked with dashed horizontal lines. c Time course data of blood glucose level by the ingestion of the glucose minimization pattern (red line and square symbols), the bolus ingestion (black solid line and plus symbols), and $1 \mathrm{~h}$-continuous (black broken line and circles). The peak values achieved for each ingestion pattern are marked with dashed horizontal lines. $\mathbf{d}$ Temporal patterns of insulin concentration simulated from ingestion of glucose according to the glucose minimization pattern (red line), insulin minimization pattern (blue line), bolus ingestion (black solid line), or $1 \mathrm{~h}$-continuous ingestion (black broken line)

minimization pattern output, we did not determine a molecular mechanism for how the intermittent pattern minimizes the peak value of blood glucose level. This question will be analyzed in the future.

We also considered the possibility that glucose minimization is because of larger insulin secretion. The peak value of insulin concentration by the glucose minimization pattern was lower than that by the bolus and 1-h-continuous ingestion. We also predicted insulin minimization pattern, and found that the intermittent pattern was optimal, which is similar to that of glucose minimization pattern. Taken together, these result suggest that the glucose minimization is achieved by suppressing the peak value of insulin concentration, rather than by enhancing insulin concentration.

Methodologically, construction of a mathematical model based on the experimental data as a forward problem is well-known in the field of the systems biology. However, the inverse problem of optimizing an input pattern to achieve a specified output pattern is challenging. ${ }^{33}$ Our success in identifying optimal input patterns through analysis of both the forward problem and inverse 
problem suggests that this approach is valid for biological systems. An obvious potential application is designing optimal ingestion patterns for various nutrients or combinations of nutrients such that the ingestion pattern that minimizes the peak value of blood glucose level can be logically designed. Such logical design of optimal food ingestion pattern will contribute the human metabolic care and the prevention of the type 2 diabetes. Here, the objective function for which we predict the input pattern is the peak value of the blood glucose level. By changing the objective function, this approach can evaluate other biological outputs and predict the input pattern that optimizes molecular concentrations or other measurable factors. Recently, a simple continuous glucose monitoring system has been available. ${ }^{35}$ If continuous glucose level together with $\mathrm{HbA} 1 \mathrm{c}$, which is a form of hemoglobin that is measured primarily to identify the threemonth average blood glucose level, ${ }^{36}$ can be measured, we can develop the predictive model of $\mathrm{HbA} 1 \mathrm{c}$ from continuous glucose blood level as a forward problem, and logically design the blood glucose patterns minimizing $\mathrm{HbA} 1 \mathrm{c}$ as an inverse problem even though the time scale is different from the current study.

Blood glucose level by the glucose minimization pattern was not so different from that by the bolus ingestion for subject \#3 in the validation experiment. Daily diet includes not only glucose, but also other nutrients such as amino acids and lipids. Therefore, the intermittent glucose ingestion pattern is not realistically useful for controlling blood glucose level. We will develop the model using the time course of blood nutrients and hormones by oral ingestion of various diet including glucose and other nutrients as a forward problem, and logically design the optimal ingestion pattern of diet that minimizes blood glucose as an inverse problem, which is practically useful, in the future.

In mathematical modeling studies in the biochemical or physiological fields, in general, the average value among subjects is often used as the experimental data fitted with the model (subject-averaged data). Even when the method of this study was applied to the subject-averaged data, the glucose minimization pattern and insulin minimization pattern were the qualitatively same intermittent pattern to the results for each subject but quantitatively different from any subject (Supplementary Fig. S4, Supplementary Tables S2-S5).

\section{Identification of individualized models of the control of blood glucose level}

Our ordinary differential equation models include the roles of incretins in insulin secretion. By determining the best fitting model for each subject, we observed differences between subjects in the roles of incretins in regulating blood glucose level. None of the subjects had models that included an independent effect of GLP-1 on insulin secretion. Two of the three subjects had no role for GLP1 (independent or cooperative with glucose) in their optimal models. In previous mathematical models using Caucasians data, only GLP-1, but not GIP, were incorporated. ${ }^{24,26}$ It has been reported that secretion of intact GLP-1 in Japanese is very small, although that of the total GLP-1 in Japanese is almost the same as that in Caucasians. ${ }^{7}$ All subjects in this study are Japanese, and, the intact GIP level was higher than the intact GLP-1 level for all of them (Fig. 2). Intact GLP-1 and intact GIP have a similar EC ${ }_{50}$ for their receptors: The $\mathrm{EC}_{50}$ of intact GIP is $8 \mathrm{nM}^{37}$ and the $\mathrm{EC}_{50}$ of intact GLP-1 is $2.6 \mathrm{nM}^{38}$ Considering the higher level of intact GIP than intact GLP-1 in the blood and their similar sensitivities, it is reasonable that intact GIP rather than intact GLP-1 was the incretin with the most effect on insulin secretion in the best fitting model. The number of subjects in this study is insufficient to conclude the presence or absence of physiological effects of incretins, and data from more subjects are needed to statistically conclude the roles of blood GLP-1 and GIP in the secretion of insulin. In addition, it is important to extend the study to pre- diabetic subjects and diabetic subjects. Although it has been reported that insulin sensitivity and insulin secretion decrease with the progression of type 2 diabetes, ${ }^{39}$ the application of this model will lead to a more precise mechanism of how changes of parameters in the model can explain the progression of insulin intolerance. Furthermore, the role of GIP and GLP-1 in the progression of insulin intolerance can be also examined. Also, it is very interesting how optimal input patterns change as progression.

Many mathematical models use average values of blood glucose from many subjects of all subjects. Some models that use data from individual subjects used data with only a single dose of glucose. ${ }^{22,28,40,41}$ Here, we used data from individual subjects using three different doses and two different durations of glucose ingestion. We constructed a mathematical model using a single dose of glucose ( $75 \mathrm{~g}$, like that of the OGTT) in subject \#1 and compared this OGTT model with the model that we constructed from the data for the three different doses and two different durations of glucose ingestion (Supplementary Fig. S5). The model that used the multiple dose and ingestion durations had a better fit to the blood glucose level achieved by ingestion of glucose according to the glucose minimization pattern (lower RSS value) than did the model using $75 \mathrm{~g}$ OGTT alone. Thus, the single dose OGTT appears insufficient to reflect the dynamics of the blood glucose level in sufficient detail for mathematical modeling, and models should be constructed from data on multiple doses and durations of glucose ingestion to be useful in predicting the glucose minimization pattern.

This finding that more training data provides more accurate predictive power is expected. However, the number of conditions for training data sets is limited in humans, because these types of studies take a long period of time and require several hours and fasting by the participants for each experimental condition. Here, we set an interval of 1-2 months for each experiment, thus collecting the data required a minimum of 6 months, and, in reality, more than a year. During such a long period, having many subjects for blood sampling following oral glucose ingestion every month over a year is difficult. Changes in the state of a subject can change during the months of the experiment, which can affect the model and reduce predictive power. Thus, in human subject tests, there is a trade-off relationship between the number of training data sets and the prediction accuracy.

A limitation of the study is that the model is limited. There are mechanisms, such as glucagon, autonomic nerves, and free fatty acids, that did not incorporate into the model. Glucagon is a counter-acting hormone to insulin in regulation of blood glucose. Glucagon increases blood glucose level by facilitating glycogenolysis. ${ }^{42,43}$ Autonomic nerves not only regulate secretion of insulin and glucagon, ${ }^{44}$ but also affect hepatic glucose production and uptake. ${ }^{4,46}$ Free fatty acids weakens the effect of insulin on hepatic glucose production and peripheral glucose uptake. ${ }^{47,48}$ Although glucagon and free fatty acid are not explicitly incorporated in our model, blood glucose levels and other hormone concentrations are well reproduced, which may suggest that the effects of other molecules such as glucagon and free fatty acid, are implicitly incorporated by some parameters in the model. Incorporating glucagon and free fatty acid explicitly into the model is a future goal.

In conclusion, the key points of this study are three. The first point is the experimental design. We performed six different conditions of oral glucose ingestion (three doses and two durations) for each subject and obtained detailed time course data, which makes the model predictable. The second point is the demonstration of ability to logically design blood glucose control. We predicted and validated the oral glucose ingestion pattern that minimized the peak value of blood glucose level. The third point is the methodology. We solved a forward problem by constructing the mathematical model of output with the given input patterns, 
and in turn, solved an inverse problem by logical designing the input pattern to control the output pattern. We expect that this approach with a forward problem and an inverse problem that are solved using the mathematical model can be widely applied to design optimal dietary ingestion pattern relevant to human health.

\section{METHODS}

Subjects

The subjects' profiles are as shown in Supplementary Table S7. All subjects are healthy, and signed informed consent.

Blood sampling experiment

For oral glucose tolerance test, a glucose solution containing 25,50 , or $75 \mathrm{~g}$ glucose was orally ingested after a 10-hour fasting, and blood samples were obtained at the times indicated in the Fig. 2 from the cutaneous vein of the forearm. Blood samples were obtained from the cutaneous vein of the forearm. Blood collection on fasting was performed twice and then a glucose solution containing 25,50 , or 75 g glucose was orally ingested. The ingestion method was rapid within a minute (bolus ingestion), and continuous over the course of $2 \mathrm{~h}$ (2-h-continuous ingestion). For continuous ingestion, we connected the tube to noncontact microdispenser robot (Mr. MJ; MECT Corporation) ${ }^{49}$ and glucose solution was ingested from tube. The subject holds the tube in the mouth and continuously ingest the glucose solution during the ingestion period. To equalize the volume of ingested glucose solution, glucose solution, TRELAN-G75 (AJINOMOTO), was diluted with distilled water into a total volume $225 \mathrm{ml}$. Each amount of glucose and delivery paradigm was tested with each subject in experiments separated by at least 1 month. Blood was rapidly centrifuged, plasma glucose and hormone concentrations expect for GIP were measured according to the methods with LSI Medience Co., Ltd. Plasma glucose was measured by enzymatic methods (IATRO LQ GLU). Plasma insulin and Serum C-peptide was measured by Chemiluminescent Immunoassay. ${ }^{50,51}$ Plasma intact GLP-1 and Plasma intact GIP were measured by ELISA kits (\#EGLP-35K, Merck, Billerica, MA or \#27201, Immuno-Biological Laboratories, Gunma, Japan, respectively). ${ }^{52,53}$ For simplicity, we refer to plasma glucose, plasma insulin, serum C-peptide, plasma intact GIP, and plasma intact GLP-1 as blood glucose, insulin, Cpeptide, GIP, and GLP-1, respectively.

\section{Validation experiment}

For the validation experiment of the glucose minimization pattern, we employed the same method as described in A.2 for the subject \#1, and a Freestyle Libre flash glucose monitoring system (FGM; Abbott Diabetes Care) for subjects \#2 and \#3. FGM reduces the invasive burden on the subjects because the subjects wear a sensor rather than requiring an indwelling needle for blood glucose monitoring. We performed the experiment after the subject had worn the sensors for at least two days. Each subject wore three sensors, and bolus ingestion, continuous ingestion for $1 \mathrm{~h}$, ingestion of glucose minimization pattern were carried out using the same sensors within 2 weeks. The results of the three sensors were averaged for each paradigm. Because FGM measures glucose level of the interstitial fluid rather than glucose level in the blood, the measured value reflects a delay of about 5-20 min (Supplementary Fig. S3) compared with the values obtained by blood collection.

\section{Ethics committee certification}

We complied with Japan's Ethical Guidelines for Epidemiological Research, and the study as approved by the ethics committees of the Life-Science Committee of the University of Tokyo (16-265). Subjects were recruited by the related law.

\section{Model structure and parameter structure}

We extended the model of Brubaker et al. ${ }^{30}$ The basic structure is the same, however, the previous models have some limitations. First, in the previous models, only single dose of bolus ingestion was used. Therefore, the previous models can not be directly applied to our study where the carious doses and temporal pattern of oral glucose ingestion were used. Second, the action of incretin is different between the previous models, and the effective action of incretin on insulin secretion has yet to be determined. Therefore by adding new differential equations for variables $A_{\text {GutG }}$ and Intest ${ }_{\mathrm{G}}\left(R_{\mathrm{GutG}}\right.$ and Duod ${ }_{\mathrm{G}}$ in Brubaker's model, respectively), we constructed multiple models of various actions of incretins on insulin secretion and selected their effective action by model selection (see below).

For each subject, we estimated parameters that reproduce the time course data of blood glucose, insulin, C-peptide, intact GIP, and intact GLP1 of six glucose ingestion patterns, combinations of three doses $(25,50$, and $75 \mathrm{~g}$ ) and given by bolus and 2-h-continuous ingestion, using the following model (Equations 1-21).

$$
\begin{aligned}
& \frac{d l i n t e s t_{6}}{d t}=v_{1}-v_{6}, \\
& \frac{d G I P}{d t}=v_{2}+v_{3}, \\
& \frac{d G L P 1}{d t}=v_{4}+v_{5}, \\
& \frac{d A_{\text {GutG }}}{d t}=v_{6}-v_{7}, \\
& \frac{d G}{d t}=\frac{v_{7}}{V}+v_{8}-v_{9}, \\
& \frac{d l}{d t}=v_{10}-v_{11}, \\
& \frac{d C P}{d t}=v_{10}-v_{12}, \\
& \frac{d X}{d t}=\frac{v_{11}}{k_{11}}-v_{13} .
\end{aligned}
$$

Equations 1-8 indicate differential equations reproducing time developments of glucose amount in the intestine Intest $G$ [g], GIP levelGIP [pM], GLP-1 levelGLP1 [pM], Absorbed amount of ingested glucose from gut $A_{\mathrm{GUtG}}[\mathrm{g}]$, blood glucose level $\left[\mathrm{mg} \mathrm{dL}^{-1}\right]$, insulin level I [pM], C-peptide $C P$ $[\mathrm{pM}]$, and the insulin level acting on the regulation of glucose $X$ (denoted as effective insulin concentration at target organs hereafter). Each variable is controlled by fluxes $v_{i}\{i=1, \cdots, 13\}$. However, in Equation $5, v_{7}$ was divided by the constant $V$ to convert the ingested glucose amount into the blood glucose level. Also in Equation 8, $v_{11}$ was divided by $k_{11}$ to render $X$ dimensionless. Rendering $X$ dimensionless enables the elimination of redundant parameters, and improves the accuracy of parameter estimation. The fluxes $v_{\mathrm{i}}$ are given by

$$
\begin{aligned}
& v_{1}=\text { Glucose } \text {, } \\
& v_{2}=\frac{k_{2} \text { Intest } t_{G}}{L_{2}+\text { Intest }}, \\
& v_{3}=k_{3}\left(G I P_{B}-G I P\right), \\
& v_{4}=\frac{k_{4} \text { Intest }_{G}}{L_{4}+\text { Intest }_{G}}, \\
& v_{5}=k_{5}\left(G L P 1_{\mathrm{B}}-G L P 1\right), \\
& v_{6}=\frac{k_{6} \text { Intest }_{G}}{L_{6}+\text { Intest }}, \\
& v_{7}=k_{7} A_{\mathrm{GutG}}, \\
& v_{8}=\frac{k_{8}}{L_{8}+X}, \\
& v_{9}=k_{9} G X, \\
& v_{10}=k_{10}(G+a G I P+b G G I P+c G L P 1+d G G L P 1), \\
& V_{11}=\frac{k_{11} l}{L_{11}+l}, \\
& v_{12}=k_{12} C P \\
& v_{13}=k_{13} X,
\end{aligned}
$$

(Figure 3). $v_{1}$ indicates the influx of ingested glucose into the intestine, given by dose of glucose ingestion divided by the time duration of ingestion $\Delta t$, otherwise 0 (Equation 22). For rapid ingestion, such as bolus ingestion, or for the ingestion of glucose minimization pattern, $\Delta t$ is assumed as 0.5 [min]. For example, in the case of $50 \mathrm{~g}$ bolus,

Glucose $=\left\{\begin{array}{c}\text { dose } / \Delta t=50 / 0.5=100,0 \leq t<0.5 \\ 0, \text { otherwise }\end{array}\right.$. 
$v_{2}$ indicates the secretion of GIP depending on the glucose amount in the intestine $\left(I n t e s t_{G}\right) . v_{3}$ indicates absorption of GIP by the intestine and entry into the blood, which is proportional to GIP subtracted by its basal $G I P_{\mathrm{B}}$. At steady state without glucose ingestion, $G I P$ converges to $G I P_{\mathrm{B}} . v_{4}$ indicates the secretion of GLP-1 depending on the glucose amount in the intestine. $v_{5}$ indicates the absorption of GLP-1 proportional to $G L P 1$ subtracted by its basal $G L P 1_{B}$. At the steady state without glucose ingestion, $G L P 1$ converges to $G L P 1_{B}$. $v_{6}$ indicates the flow of glucose adsorption $\left(A_{\text {GutG }}\right)$. With bolus ingestion, this flow can be regarded as constant because of the large amount of glucose in the intestine. ${ }^{30}$ Therefore, we assumed that this flux is given by the Michaelis-Menten equation, which saturates when the glucose amount is large. $v_{7}$ indicates the flow of glucose from the rate of appearance into the blood, which is proportional to the rate of appearance of ingested glucose amount. $v_{8}$ indicates the flow of glucose production from the liver into the blood, given by an inhibitory Michaelis-Menten equation, which decreases as the amount of effective insulin $X$ increases. $v_{9}$ indicates the glucose uptake from the blood to the periphery and is given by the product between blood glucose level $G$ and effective insulin $X . v_{10}$ indicates the secretion of insulin. In this study, the actions of GIP and GLP-1 on insulin secretion were represented as independent actions of each incretin and as cooperative actions with blood glucose. By incorporating the parameters $(a, b, c$, and $d$ in Equation 18), we could relate insulin secretion to cooperative or independent actions using AIC (Akaike Information Criteria) to select the model that best fit the data (Supplementary Tables S1, S2). $v_{11}$ indicates the flow of insulin / into target organs, such as liver and muscle, leading to effective insulin $X . v_{12}$ indicates inactivation of C-peptide $C P$ and decreases in proportion to $C P$ itself. $v_{13}$ indicates the binding of $X$ to the cells in the target organs in proportion to $X$ itself.

For the model, parameters were estimated for each subject. Here, the estimated parameters are the 18 parameters of $k_{2}, k_{3}, k_{4}, k_{5}, k_{6}$, $k_{7}, k_{8}, k_{10}, L_{2}, L_{4}, L_{6}, L_{8}, L_{11}, V, a, b, c$ and $d$; and six initial levels of $G I P(0), G L P 1(0), G(0), I(0), C P(0)$ and $X(0)$. Using the variables, and assuming Intest ${ }_{G}, A_{G u t G}, G I P, G L P 1, G, I, X$, and $C P$ are at steady state before ingestion, other initial conditions and parameters were determined by estimated parameters and initial values, given by

Ingest $_{\mathrm{G}}(0)=0$,

$A_{\text {GutG }}(0)=0$,

$k_{9}=\frac{k_{8}}{G(0) \cdot X(0) \cdot\left(X(0)+L_{8}\right)}$,

$k_{11}=k_{10} \frac{L_{11}+l(0)}{I(0)}(G(0)+a G I P(0)+b G(0) G I P(0)+c G L P 1(0)+d G(0) G L P 1(0)$

$k_{12}=\frac{k_{10}}{C P(0)}(G(0)+a G I P(0)+b G(0) G I P(0)+c G L P 1(0)+d G(0) G L P 1(0))$,

$k_{13}=\frac{l(0)}{\left(I(0)+L_{11}\right) X(0)}$,

$G I P_{\mathrm{B}}=G I P(0)$,

$G L P 1_{\mathrm{B}}=G L P 1(0)$.

These parameters are different between subjects, but the same for each subject for each experimental paradigm (dose and duration and ingestion). This means that the state for each subject does not change during this study. Note that the time courses by $75 \mathrm{~g} \mathrm{2-h}$ continuous ingestion for the subject \#2 were excluded for the parameter estimation because the subject's condition and the surrounding environment have changed significantly between this and the other experiments. For time development, we used CVODE in Matlab's Systems biology toolbox (SBToolbox2).

We used the residual sum of squares (RSS) as the objective function so that the residual between the experimental value and the simulation value is reduced, given by

$\mathrm{RSS}=\sum_{i} \sum_{k} \sum_{t}\left[\frac{x_{i, k}^{\operatorname{sim}}(t)-x_{i, k}^{\exp }(t)}{\max _{t} x_{i, k}^{\text {exp }}(t)-\min _{t} x_{i, k}^{\exp }(t)}\right]^{2}$.

$x_{i, k}^{\text {sim }}(t)$ and $x_{i, k}^{\exp }(t)$ indicate the simulation values and the experimental values of molecular species $k \in\{G, I, C P, G L P 1, G I P\}$ at time $t$ in the experiment $i \in\{25 \mathrm{~B}, 25 \mathrm{C}, 50 \mathrm{~B}, 50 \mathrm{C}, 75 \mathrm{~B}, 75 \mathrm{C}\}$, for which each experiment is denoted by the ingestion dose and the initial letters of the duration of ingestion, 25-g-bolus ingestion, 25B and for 75-g-2-h-continuous, 75 C. To avoid the influences of the differences in the absolute quantities of the molecules, we normalized the difference between the simulation value and the experimental value by the difference between the maximum value and the minimum value of the experiment. We performed parameter estimation for global optimal solution using Evolutionary programming ${ }^{34}$ for 40 trials with a parent number of 5000 and a generation number of 5000, then we obtained a local optimal solution using the simplex search method (Matlab fminsearch). We implemented all programs using Matlab 2015a and performed parameter estimation using $2.6 \mathrm{GHz}$ CPU (Xeon E5 2670) at the National Institute of Genetics (NIG), Supercomputer System of Research Organization of Information and System (ROIS).

\section{Model selection}

Using parameters of $a, b, b$ and $d$ in Equation 18, which indicate contributions to insulin secretion of incretins as independent actions of each incretin and cooperative actions with glucose, we considered the multiple models shown in Supplementary Table S1.

We performed the parameter estimation of each of the above models using RSS of Equation 31 for each subject. Here, we assumed that each residual of the simulation value and the experiment value in Equation 31 follows a normal distribution. Among the models to be compared, the sum $N$ of the numbers of data of each variable measured in the experiment is the same. Therefore, AIC (Akaike Information Criteria), which is a criterion of model selection can be calculated for each model, given by

$\mathrm{AIC}=N \log (\mathrm{RSS})+2 K$.

We employed a model that minimizes AIC for each subject as a model representing the dynamics of blood molecules in the subject. For the models not including GLP-1 of subjects \#1 and \#3 as mentioned below, we also calculated AIC for each model similar to those including GLP-1.

The selected models for each subject were distinct (Table 2, Supplementary Tables S1, S2). For subject \#1, the best model had no influence of GLP-1 and both an independent action and cooperative action with glucose for GIP (Supplementary Table S2, $c=d=0$ ), indicating that the insulin secretion of subject \#1 is independent of GLP-1. For subject \#2, the best model had an independent action of GIP and a cooperative action of GLP-1 with glucose (Supplementary Table $S 2, b=c=0$ ), indicating that the insulin secretion of subject \#2 depends on both GIP and GLP-1. For subject \#3, the best model had only the cooperative action of GIP with glucose (Supplementary Table S2, $\mathrm{a}=\mathrm{c}=\mathrm{d}=0$ ), indicating that the insulin secretion of subject \#3 is independent of GLP-1. In each subject model, time course data of each blood glucose and hormones were approximately reproduced (Fig. 3b, Supplementary Figs. S2 and S3).

In the selected models of subjects \#1 and \#3, insulin secretion did not depend on GLP-1, therefore, we performed parameter estimation and model selection using models that did not include GLP-1 by removing Equation 3. Insulin secretion using the best fitting of these models for both subjects \#1 and \#3 included the term of independent action of blood glucose and the cooperative term of blood glucose and GIP (Supplementary Table S3, $a=0$ ). We used these models for subjects \#1 and \#3.

\section{Estimation of minimization patterns}

We set the oral glucose $u(t)$ as a function of time $t$ [min] according to the following constraint condition. First, glucose was orally ingested at intervals of $5 \mathrm{~min}$ from $0 \mathrm{~min}$ to $60 \mathrm{~min}$ Here, we defined $u_{s}[\mathrm{~g}]$ as the dose of ingestion at the minute $s[\mathrm{~min}](s=0,5, \ldots, 60)$ and $\mathbf{u}_{0: 60}$ as the temporal pattern of oral glucose ingestion, given by

$\mathbf{u}_{0: 60}=\left[u_{0}, u_{5}, \ldots, u_{60}\right]$

Also, we set the total dose of glucose ingestion at $50 \mathrm{~g}$, i.e., $\sum u_{s}=50$ [g], each dose at $s$ is the integer value with unit of $1 \mathrm{~g}$, i.e., $u_{s} \in \mathbb{Z}, u_{s} \geq 0$, and at least $1 \mathrm{~g}$ is ingested at $0 \mathrm{~min}$ to start the ingestion, i.e., $u_{0} \geq 1$. We assumed that ingestion at each time is taken over $0.5 \mathrm{~min}$, and convert $\mathbf{u}_{0: 60}$ to Glucose instead of Equation 9, given by

Glucose $(t)=\left\{\begin{array}{c}u_{s} / 0.5 \quad t_{i} \leq t<t_{i}+0.5, \quad t_{i} \in\{0,5, \cdots, 60\} \\ 0 \text { otherwise }\end{array}\right.$.

Next, we expressed a nonlinear ordinary differential equation model (Equations 1-8) describing the dynamics of the glucose metabolism 
system, given by

$$
\frac{d \mathbf{x}}{d t}=f(u(t), \mathbf{x}(t) ; \boldsymbol{\theta}),
$$

$x(0)=\mathbf{x}^{\text {init }}$,

where $\mathbf{x}$ indicates a state variable, $\mathbf{x}^{\text {init }}$ indicates an initial state, $\boldsymbol{\theta}$ is a parameter set, and $\mathbf{f}$ is a nonlinear function. These types and values of $\mathbf{x}$, $\mathbf{x}^{\text {init }}, \boldsymbol{\theta}$, and $\mathbf{f}$ are different among subjects, because the selected models of subjects and parameters are different among subjects (see above). Each subject has one set of $\mathbf{f}, \mathbf{x}^{\text {init }}$, and $\boldsymbol{\theta} . \mathbf{x}(0: T)$, the temporal pattern of $\mathbf{x}$ from $t=0$ to $t=T$ with the temporal pattern of oral glucose ingestion $\mathbf{u}_{0: 60}$, can be obtained by the deterministic numerical simulation of this mathematical model Sim, given by

$x(0: T)=\operatorname{Sim}\left(\mathbf{u}_{0: 60}, \mathbf{x}^{\text {init }}, \boldsymbol{\theta}, \mathbf{f}, T\right)$.

To design a temporal pattern of oral glucose ingestion that minimizes the peak value of blood glucose level or insulin concentration, we formulated as an optimization problem. Defining the peak values of blood glucose level and insulin concentration in the time course $\mathbf{x}(0: T)$ as $G_{\operatorname{Max}}(\mathbf{x}(0: T))$ and $I_{\operatorname{Max}}(\mathbf{x}(0: T))$, respectively, and setting the objective function of the optimization problem to be $J(\mathbf{x}(0: T))$, we set the objective functions for designing the temporal patterns of oral glucose ingestion that minimizes the peak value of blood glucose level or insulin concentration, given by

$J(\mathbf{x}(0: T))=\left\{\begin{array}{c}G_{\operatorname{Max}}(\mathbf{x}(0: T)) \text { for glucose minimization pattern } \\ I_{\operatorname{Max}}(\mathbf{x}(0: T)) \text { for insulin minimization pattern }\end{array}\right.$

Under these settings, the optimization problem of designing the oral glucose ingestion pattern can be expressed as follows for minimizing the peak value of blood glucose level, given by

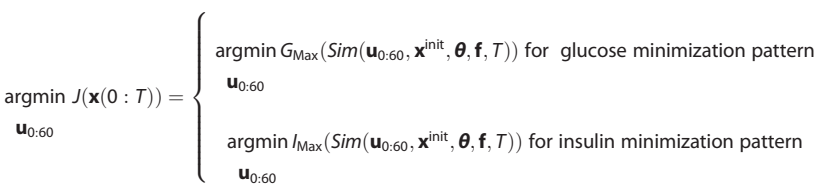

We numerically solved this optimization problem by following evolutionary programming. Each individual has an oral glucose ingestion pattern. After initialization of the oral glucose ingestion pattern of each individual, the algorithm outputs the oral glucose ingestion pattern that minimizes the objective function value by repeating (i) the mutation steps through which a new oral glucose ingestion pattern for each individual is proposed, and (ii) the selection steps through which individual (and thus new pattern) are selected based on the value of the objective function.

Denoting the total number of individuals as $N$, the $n^{\text {th }}$ individual of the oral glucose ingestion pattern $\mathbf{u}_{0: 60}$ as $\mathbf{u}_{n}$, and simplifying the objective function as $J\left(\mathbf{u}_{n}\right)$, the algorithm is as follows:

(1) (Initialization) For each individual $n=1, \ldots, N, \mathbf{u}_{n}$ is initialized and $\mathbf{u}_{n}$ that minimizes $J\left(\mathbf{u}_{n}\right)$ is stored as $\mathbf{u}^{*}$.

(2) Repeat the following procedure (a)-(c) $K$ times

a. (Mutation) For each individual $(n=1, \ldots, N)$, copy and mutate $\mathbf{u}_{n}$ to generate a new individual $\mathbf{u}_{n}^{\prime}$. Update $\mathbf{u}^{*}$ as $\mathbf{u}^{*} \leftarrow \mathbf{u}_{n}^{\prime}$ if $J\left(\mathbf{u}^{*}\right)>J\left(\mathbf{u}_{n}^{\prime}\right)$.

b. (Selection 1) For each of $2 N$ individuals that consist of the original individuals and the new individuals generated at (a), obtain the evaluation value by the following procedure.

i. Select an individual sequentially as $\mathbf{u}_{m}$.

ii. Select an $M$ individuals randomly except for $\mathbf{u}_{m}$ (duplication possible) as $\mathbf{u}_{m_{i}}(i=1, \ldots, M)$.

iii. Obtain the evaluation value defined by the number of $\mathbf{u}_{m_{i}}$ with $J\left(\mathbf{u}_{m_{i}}\right)>J\left(\mathbf{u}_{m}\right)$.

c. (Selection 2) Sort the individuals in order of the evaluation value, and the top $N$ individuals are selected and used in the next step.

(3) Output $\mathbf{u}^{*}$

In terms of evolutionary programming, step 1 is initialization, step 2-a is mutation, and steps 2-b and 2-c are selection. Because the intersection of oral glucose ingestion patterns is complicated by the constraint of $50 \mathrm{~g}$ total ingestion dose, this algorithm does not include intersection.
Details of initialization and mutation are as follows: In initialization, to avoid bias of an initial value, $N$ individuals consist of an individual with a $50 \mathrm{~g}$ bolus ingestion, an individual with $1 \mathrm{~g}$ ingestion at $0 \mathrm{~min}$ and the remaining $49 \mathrm{~g}$ ingestion at $60 \mathrm{~min}$, and other random patterns. The random pattern was generated by distributing $49 \mathrm{~g}$ glucose randomly with equal probability at each time point and the remaining $1 \mathrm{~g}$ ingestion at 0 min For the mutation, a new oral glucose ingestion pattern was suggested by repeating operations that transfer $1 \mathrm{~g}$ of glucose from one time point to another randomly. Specific operations are as follows.

1. Subtract $1 \mathrm{~g}$ of glucose at time $0 \mathrm{~min}$

2. Repeat the following procedure (a) and (b) $L$ times

a. Randomly select the source and destination time points of glucose with equal probability.

b. If the ingestion glucose at the source time point contains more than $1 \mathrm{~g}$, transfer $1 \mathrm{~g}$ of glucose from the source time point to the destination time point.

3. Add $1 \mathrm{~g}$ of glucose at time $0 \mathrm{~min}$

In the deterministic numerical simulation Sim, we employed the Euler method with a time step width of 0.001 [min] to shorten the calculation time. We also set $T=480$ [min].

In the evolutionary programming, we set the number of individuals as $N=500$, the number of generation except initialization generation as $K=$ 500 , the number of transfers of glucose in one mutation $L$ to decrease from $L=20$ by 1 every 25 generations, and the number of individuals for calculation of evaluation value in selection as $M=N / 5=100$. According to this algorithm and these settings, we calculated the optimal ingestion pattern for five trials and obtained the pattern that produced the smallest objective function. Note that we obtained the same minimization pattern for each subject multiple times for multiple trials (all trials in subject \#1 and \#2, 2 trials in subject \#3).

Reporting summary

Further information on research design is available in the Nature Research Reporting Summary linked to this article.

\section{DATA AVAILABILITY}

All data generated or analysed during this study are included in this published article and its supplementary materials files. The SBToolbox2 (http://www.sbtoolbox2.org/ main.php) was used together with Matlab R2015a (http://mathworks.com/) in the mathematical modelling.

\section{CODE AVAILABILITY}

The code files used in the simulation are freely available at http://kurodalab.org/ publication/info/fujii.

\section{ACKNOWLEDGEMENTS}

We thank fellow laboratory members for critical reading of the manuscript and for technical assistance with the experiment and analysis. We thank Rika Sumita, Mina Shiguma, and Naomi Isene for technical assistance. We thank Nancy R. Gough (BioSerendipity, LLC) for editing the manuscript. The computations for this work were performed in part on the NIG supercomputer system at ROIS National Institute of Genetics. This work was supported by the Creation of Fundamental Technologies for Understanding and Control of Biosystem Dynamics (JPMJCR12W3), CREST, of the Japan Science and Technology Agency (JST) and by the Japan Society for the Promotion of Science (JSPS) KAKENHI Grant Number (17H06300, 17H6299, 18H03979, 19K22860). M.F. receives funding from a Grant-in-Aid for Challenging Exploratory Research (16K12508) and a Grant-in-Aid for Young Scientists (19K20382). Y.K. receives funding from a Grant-in-Aid for Young Scientists (18K16578). S.U. receives funding from Kayamori Foundation of Informational Science Advancement and a Grant-in-Aid for Scientific Research on Innovative Areas (18H04801). H.K. receives funding from a Grant-in-Aid for Scientific Research on Innovative Areas (16H06577). K.K. receives funding from a Grant-in-Aid for Scientific Research (B) (15KT0021) and (C) (15K00246), and a Grant-in-Aid for Scientific Research on Innovative Areas (16H01554). The funders had no role in study design, data collection and analysis, decision to publish, or preparation of the manuscript. 


\section{AUTHOR CONTRIBUTIONS}

M.F., Y.M., S.O., and S.K. conceived the project. Y.K., Y.S., and S.F. performed the experiment. M.F. and Y.M. constructed the model and performed the simulation. M.F., Y.M., M.K., S.U., H.K., H.I, K.K., S.O., S.I,, and S.K. analysed the data. M.F., Y.M., Y.K., and S. K. wrote the manuscript. S.I. and S.K. supervised.

\section{ADDITIONAL INFORMATION}

Supplementary Information accompanies the paper on the npj Systems Biology and Applications website (https://doi.org/10.1038/s41540-019-0108-1).

Competing interests: The authors declare no competing interests.

Publisher's note: Springer Nature remains neutral with regard to jurisdictional claims in published maps and institutional affiliations.

\section{REFERENCES}

1. Abdul-Ghani, M. A., Tripathy, D. \& DeFronzo, R. A. Contributions of beta-cell dysfunction and insulin resistance to the pathogenesis of impaired glucose tolerance and impaired fasting glucose. Diabetes Care 29, 1130-1139 (2006).

2. Edelstein, S. L. et al. Predictors of progression from impaired glucose tolerance to NIDDM: an analysis of six prospective studies. Diabetes 46, 701-710 (1997).

3. Nakagami, T. \& DECODA Study Group. Hyperglycaemia and mortality from all causes and from cardiovascular disease in five populations of Asian origin. Diabetologia 47, 385-394 (2004).

4. Cahill, G. F. Fuel metabolism in starvation. Annu. Rev. Nutr. 26, 1-22 (2006).

5. Schulze, M. B. et al. Glycemic index, glycemic load, and dietary fiber intake and incidence of type 2 diabetes in younger and middle-aged women. Am. J. Clin. Nutr. 80, 348-356 (2004).

6. Castillo, M. J., Scheen, A. J., Letiexhe, M. R. \& Lefèbvre, P. J. How to measure insulin clearance. Diabetes Metab. Rev. 10, 119-150 (1994).

7. Seino, Y., Fukushima, M. \& Yabe, D. GIP and GLP-1, the two incretin hormones: Similarities and differences. J. Diabetes Investig. 1, 8-23 (2010).

8. Vollmer, K. et al. Predictors of incretin concentrations in subjects with normal, impaired, and diabetic glucose tolerance. Diabetes 57, 678-687 (2008).

9. Fujimoto, W. et al. Niflumic acid-sensitive ion channels play an important role in the induction of glucose-stimulated insulin secretion by cyclic AMP in mice. Diabetologia 52, 863-872 (2009).

10. Preitner, F. et al. Gluco-incretins control insulin secretion at multiple levels as revealed in mice lacking GLP-1 and GIP receptors. J. Clin. Invest. 113, 635-645 (2004).

11. Inagaki, N. et al. Gastric inhibitory polypeptide: structure and chromosomal localization of the human gene. Mol. Endocrinol. 3, 1014-1021 (1989).

12. Takeda, J. et al. Sequence of an intestinal cDNA encoding human gastric inhibitory polypeptide precursor. Proc. Natl Acad. Sci. USA 84, 7005-7008 (1987).

13. Orskov, C., Wettergren, A. \& Holst, J. J. Biological effects and metabolic rates of glucagonlike peptide-1 7-36 amide and glucagonlike peptide-1 7-37 in healthy subjects are indistinguishable. Diabetes 42, 658-661 (1993).

14. Bell, G. I., Santerre, R. F. \& Mullenbach, G. T. Hamster preproglucagon contains the sequence of glucagon and two related peptides. Nature 302, 716-718 (1983).

15. Parkes, D. G., Pittner, R., Jodka, C., Smith, P. \& Young, A. Insulinotropic actions of exendin-4 and glucagon-like peptide- 1 in vivo and in vitro. Metabolism 50, 583-589 (2001).

16. Stumvoll, M. et al. Use of the oral glucose tolerance test to assess insulin release and insulin sensitivity. Diabetes Care 23, 295-301 (2000).

17. Dalla Man, C., Camilleri, M. \& Cobelli, C. A system model of oral glucose absorption: validation on gold standard data. IEEE Trans. Biomed. Eng. 53, 2472-2478 (2006).

18. Dalla Man, C., Raimondo, D. M., Rizza, Ra \& Cobelli, C. GIM, Simulation Software of Meal Glucose-Insulin Model. J. Diabetes Sci. Technol. 1, 323-330 (2007).

19. Salinari, S., Bertuzzi, A. \& Mingrone, G. Intestinal transit of a glucose bolus and incretin kinetics: a mathematical model with application to the oral glucose tolerance test. Am. J. Physiol. Endocrinol. Metab. 300, E955-E965 (2011).

20. Hill, K., Caprihan, A. \& Kakalios, J. Bulk segregation in rotated granular material measured by magnetic resonance imaging. Phys. Rev. Lett. 78, 50-53 (1997).

21. Røge, R. M. et al. Mathematical modelling of glucose-dependent insulinotropic polypeptide and glucagon-like peptide-1 following ingestion of glucose. Basic Clin. Pharmacol. Toxicol. 38, 42-49 (2017).

22. Dalla Man, C. et al. Modeling hepatic insulin sensitivity during a meal: validation against the euglycemic hyperinsulinemic clamp. Am. J. Physiol. Endocrinol. Metab. 304, E819-E825 (2013).

23. Overgaard, R. V., Jelic, K., Karlsson, M., Henriksen, J. E. \& Madsen, H. Mathematical beta cell model for insulin secretion following IVGTT and OGTT. Ann. Biomed. Eng. 34, 1343-1354 (2006)
24. Pedersen, M. G., Dalla Man, C. \& Cobelli, C. Multiscale modeling of insulin secretion. IEEE Trans. Biomed. Eng. 58, 3020-3023 (2011).

25. Riz, M. et al. Minimal modeling of insulin secretion in the perfused rat pancreas: a drug effect case study. Am. J. Physiol. Endocrinol. Metab. 306, E627-E634 (2014).

26. Dalla Man, C., Micheletto, F., Sathananthan, M., Vella, A. \& Cobelli, C. Model-based quantification of glucagon-like peptide-1-induced potentiation of insulin secretion in response to a mixed meal challenge. Diabetes Technol. Ther. 18, 39-46 (2016).

27. Tura, a, Ludvik, B., Nolan, J. J., Pacini, G. \& Thomaseth, K. Insulin and C-peptide secretion and kinetics in humans: direct and model-based measurements during OGTT. Am. J. Physiol. Endocrinol. Metab. 281, E966-E974 (2001).

28. De Gaetano, A. et al. Routine OGTT: a robust model including incretin effect for precise identification of insulin sensitivity and secretion in a single individual. PLOS ONE 8, e70875 (2013).

29. Bergman, R. N., Ider, Y. Z., Bowden, C. R. \& Cobelli, C. Quantitative estimation of insulin sensitivity. Am. J. Physiol. 236, E667-E677 (1979).

30. Brubaker, P. L., Ohayon, E. L., D’Alessandro, L. M. \& Norwich, K. H. A mathematical model of the oral glucose tolerance test illustrating the effects of the incretins. Ann. Biomed. Eng. 35, 1286-1300 (2007).

31. Kim, M. et al. Simulation of oral glucose tolerance tests and the corresponding isoglycemic intravenous glucose infusion studies for calculation of the incretin effect. J. Korean Med. Sci. 29, 378-385 (2014).

32. Kabul, R. S. E. et al. Mathematical model of glucose-insulin system using the modified oral minimal model and the incretin effects. Int. J. Pharm. Pharm. Sci. 7, 451-454 (2015).

33. Murakami, Y., Koyama, M., Oba, S., Kuroda, S. \& Ishii, S. Model-based control of the temporal patterns of intracellular signalingin silico. Biophys. Phys. 14, 29-40 (2017).

34. Bäck, T. \& Schwefel, H.-P. An overview of evolutionary algorithms for parameter optimization. Evol. Comput. 1, 1-23 (1993).

35. Bailey, T., Bode, B. W., Christiansen, M. P., Klaff, L. J. \& Alva, S. The performance and usability of a factory-calibrated flash glucose monitoring system. Diabetes Technol. Ther. 17, 787-794 (2015).

36. Rohlfing, C. L. et al. Defining the relationship between plasma glucose and $\mathrm{HbA}$ (1c): analysis of glucose profiles and $\mathrm{HbA}(1 \mathrm{c})$ in the Diabetes Control and Complications Trial. Diabetes Care 25, 275-278 (2002).

37. Gespach, C., Emami, S. \& Rosselin, G. Gastric inhibitory peptide (GIP), pancreatic glucagon and vasoactive intestinal peptide (VIP) are CAMP-inducing hormones in the human gastric cancer cell line HGT-1. Homologous desensitization of VIP receptor activity. Biochem. Biophys. Res. Commun. 120, 641-649 (1984).

38. Adelhorst, K., Hedegaard, B. B., Knudsen, L. B. \& Kirk, O. Structure-activity studies of glucagon-like peptide-1. J. Biol. Chem. 269, 6275-6278 (1994).

39. Schofield, C. J. \& Sutherland, C. Disordered insulin secretion in the development of insulin resistance and Type 2 diabetes. Diabet. Med. 29, 972-979 (2012)

40. Ohashi, K. et al. Glucose homeostatic law: insulin clearance predicts the progression of glucose intolerance in humans. PLOS ONE 10, e0143880 (2015).

41. Ohashi, K. et al. Increase in hepatic and decrease in peripheral insulin clearance characterize abnormal temporal patterns of serum insulin in diabetic subjects. npj Syst. Biol. Appl. 4, 14 (2018).

42. Jiang, G. \& Zhang, B. B. Glucagon and regulation of glucose metabolism. Am. J. Physiol. Endocrinol. Metab. 284, E671-E678 (2003).

43. Alberti, K. G. \& Zimmet, P. Z. Definition, diagnosis and classification of diabetes mellitus and its complications. Part 1: diagnosis and classification of diabetes mellitus provisional report of a WHO consultation. Diabet. Med. 15, 539-553 (1998).

44. Thorens, B. Brain glucose sensing and neural regulation of insulin and glucagon secretion. Diabetes Obes. Metab. 13(Suppl 1), 82-88 (2011).

45. Ruud, J., Steculorum, S. M. \& Brüning, J. C. Neuronal control of peripheral insulin sensitivity and glucose metabolism. Nat. Commun. 8, 15259 (2017).

46. Kimura, K. et al. Central insulin action activates kupffer cells by suppressing hepatic vagal activation via the nicotinic alpha 7 acetylcholine receptor. Cell Rep. 14, 2362-2374 (2016).

47. Okuno, A. et al. Troglitazone increases the number of small adipocytes without the change of white adipose tissue mass in obese Zucker rats. J. Clin. Invest. 101, 1354-1361 (1998).

48. Yamauchi, T. et al. The mechanisms by which both heterozygous peroxisome proliferator-activated receptor gamma (PPARgamma) deficiency and PPARgamma agonist improve insulin resistance. J. Biol. Chem. 276, 41245-41254 (2001).

49. Sano, T. et al. Selective control of up-regulated and down-regulated genes by temporal patterns and doses of insulin. Sci. Signal. 9, ra112 (2016).

50. Tietz, N. W., Finley, P. R. \& Pruden, E. L. Clinical Guide to Laboratory Tests, 2nd Edition. (WB Saunders, Philadelphia, 1990).

51. Tholen, D. W., Kallner, A. J., Kennedy, W., Krouwer, J. S. \& Meier, K. Evaluation of Precision Performance of Quantitative Measurement Methods; Approved Guideline. 
2nd edition. 24, No. 25, EP05-A2. (National Committee on Clinical Laboratory Standards, Pennsylvania, 2004).

52. Tijssen, P. Practice and theory of enzyme immunoassays. (Elsevier, Amsterdam, 1985).

53. Miyawaki, K. et al. Inhibition of gastric inhibitory polypeptide signaling prevents obesity. Nat. Med. 8, 738-742 (2002)
Open Access This article is licensed under a Creative Commons Attribution 4.0 International License, which permits use, sharing, adaptation, distribution and reproduction in any medium or format, as long as you give appropriate credit to the original author(s) and the source, provide a link to the Creative Commons license, and indicate if changes were made. The images or other third party material in this article are included in the article's Creative Commons license, unless indicated otherwise in a credit line to the material. If material is not included in the article's Creative Commons license and your intended use is not permitted by statutory regulation or exceeds the permitted use, you will need to obtain permission directly from the copyright holder. To view a copy of this license, visit http://creativecommons. org/licenses/by/4.0/.

(c) The Author(s) 2019 